\title{
Hitting probabilities for non-convex lattice
}

\author{
G. Caristi ${ }^{\mathrm{a}, *}$, M. Pettineo ${ }^{\mathrm{b}}$, A. Puglisi ${ }^{\mathrm{a}}$ \\ ${ }^{a}$ Department of Economics, University of Messina, via dei Verdi, 75 98122, Messina, Italy. \\ ${ }^{b}$ Department of Mathematics, University of Palermo, via Archirafi, 34-Palermo, Italy.
}

Communicated by C. Vetro

\begin{abstract}
In this paper, we consider three lattices with cells represented in Figures 1, 3, and 5 and we determine the probability that a random segment of constant length intersects a side of the lattice considered.
\end{abstract}

Keywords: Geometric probability, stochastic geometry, random sets, random convex sets and integral geometry.

2010 MSC: 60D05, 52A22.

(C)2018 All rights reserved.

\section{Introduction}

Caristi and Stoka [7] and [8] introduced in the Buffon-Laplace type problems so-called obstacles. They considered two lattices with axial symmetry and in a first moment [7] they study with eight triangular and circular sector obstacles and in the second moment [8] they analyze twelve obstacles. Several other authors considered different lattices with different types of obstacles and studied the probability that a random body test intersect the fundamental cell [2,5], and [4]. In particular, in [1], the authors studied a Laplace type problem with obstacles for two Delone hexagonal lattices and in [6] for a regular lattice of Dirichlet-Voronoi. In this study, starting from the results obtained by Duma and Stoka [9] for Buffon type problems with a nonconvex lattice we consider a Laplace type problem for three lattices with triangular obstacles, circular sector obstacles and triangular and sectors circular together. We study the probability that a random segment of constant length intersects the fundamental cells in Figures 1, 3, and 5.

\section{Obstacles triangular}

Let $\Re_{1}(a, b ; m)$ be the lattice with the fundamental cell $C_{1}$ represented in Figure 1 , where $a<b$ and $\mathrm{m}<\mathrm{a} / 2$. From Figure 1 we have

$$
\operatorname{areaC}_{1}=3 a b-\frac{5}{2} m^{2}
$$

We compute the probability that a random segment $s$ of constant length $l<\frac{a}{2}-m$ intersects a side of lattice $\mathfrak{R}_{1}$, i.e., the probability $\mathrm{P}_{\mathrm{int}}^{(1)}$ that the segment $\mathrm{s}$ intersects a side of fundamental cell $\mathrm{C}_{1}$.

The position of segment $s$ is determined by its center and by the angle $\varphi$ that it formed with the side $B C$ (or AF) of the cell $C_{1}$.

\section{*Corresponding author}

Email addresses: gcaristi@unime.it (G. Caristi), maria.pettineo@unipa.it (M. Pettineo), puglisia@unime.it (A. Puglisi)

doi: $10.22436 /$ jnsa.011.04.05

Received: 2017-08-01 Revised: 2017-09-29 Accepted: 2017-11-21 
To compute $P_{i n t}$ we consider the limiting positions of segment $s$, for a fixed angle of $\varphi$, in the cell $C_{1}$. We obtain the Figure 2

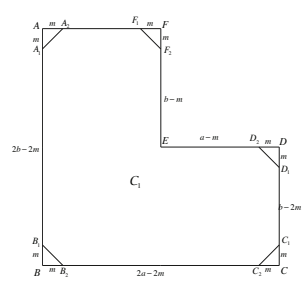

Figure 1

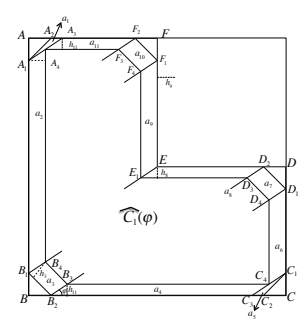

Figure 2

and the formula

$$
\operatorname{area} \widehat{C}_{1}(\varphi)=\operatorname{areaC}_{1}-\sum_{i=1}^{11} \operatorname{aread}_{i}(\varphi) .
$$

Theorem 2.1. We have

$$
P_{\text {int }}^{(1)}=\frac{2\left[2(a+b) l-\frac{l^{2}}{2}-\frac{\pi}{2} m^{2}\right]}{\pi\left(3 a b-\frac{5}{2} m^{2}\right)}
$$

Proof. By Figure 2 we have

$$
\begin{array}{rlrl}
\operatorname{areaAA}_{1} A_{3} & =\frac{\mathrm{ml}}{2} \cos \varphi, & \operatorname{areaa}_{1}(\varphi) & =\operatorname{areaa}_{5}(\varphi)=\frac{\mathrm{ml}}{2} \cos \varphi-\frac{\mathrm{m}^{2}}{2}, \\
\operatorname{areaa}_{2}(\varphi) & =(\mathrm{b}-\mathrm{m}) l \cos \varphi, & \operatorname{areaa}_{11}(\varphi)=\frac{\mathrm{al}}{2} \sin \varphi-\frac{\mathrm{ml}}{2} \sin \varphi-\frac{l^{2}}{4} \sin 2 \varphi, \\
\operatorname{areaa}_{6}(\varphi) & =\frac{\mathrm{bl}}{2} \cos \varphi-m l \cos \varphi, & \operatorname{areaa}_{3}(\varphi)=\operatorname{areaa}_{7}(\varphi)=\operatorname{aread}_{10}(\varphi)=\frac{\mathrm{ml}}{2}(\sin \varphi+\cos \varphi), \\
\operatorname{areaa}_{4}(\varphi)=\mathrm{al} \sin -\frac{\mathrm{ml}}{2} \sin \varphi-\frac{l^{2}}{4} \sin 2 \varphi, & \operatorname{areaa}_{8}(\varphi)=\frac{\mathrm{al}}{2} \sin \varphi-\frac{\mathrm{ml}}{2} \sin \varphi, \\
\operatorname{areaa}_{9}(\varphi)=\frac{\mathrm{bl}}{2} \cos \varphi-\frac{\mathrm{ml}}{2} \cos \varphi . &
\end{array}
$$

We can write that

$$
A_{1}(\varphi)=\sum_{i=1}^{11} \operatorname{areaa}_{i}(\varphi)=2 a l s i n \varphi+2 b l c o s \varphi-\frac{l^{2}}{2} \sin 2 \varphi-m^{2} .
$$

Replacing this relation in formula (2.2) follows

$$
\operatorname{areaC}_{1}(\varphi)=\operatorname{areaC}_{1}-A_{1}(\varphi) .
$$

We denote with $M_{1}$, the set of segments $s$ that they have center in the cell $C_{1}$, and with $N_{1}$ the set of segments $s$ entirely contained in the cell $C_{1}$, so we have [11],

$$
P_{\text {int }}^{(1)}=1-\frac{\mu\left(N_{1}\right)}{\mu\left(M_{1}\right)}
$$

where $\mu$ is the Lebesgue measure in the euclidean plane.

To compute the measure $\mu\left(M_{1}\right)$ and $\mu\left(N_{1}\right)$ we use the kinematic measure of Poincarè [10]:

$$
\mathrm{dk}=\mathrm{d} x \wedge \mathrm{dy} \wedge \mathrm{d} \varphi,
$$

where $x, y$ are the coordinate of center of $s$ and $\varphi$ the fixed angle.

For $\varphi \in\left[0, \frac{\pi}{2}\right]$ we have

$$
\mu\left(M_{1}\right)=\int_{0}^{\frac{\pi}{2}} d \varphi \iint_{\left\{(x, y) \in C_{1}\right\}} d x d y=\int_{0}^{\frac{\pi}{2}}\left(\text { area }_{1}\right) d \varphi=\frac{\pi}{2} \operatorname{areaC}_{1} .
$$

In the same way, considering formula (2.4) we can write 


$$
\begin{aligned}
\mu\left(\mathrm{N}_{1}\right) & =\int_{0}^{\frac{\pi}{2}} \mathrm{~d} \varphi \iint_{\left\{(x, y) \in C_{1(\varphi)}\right\}} \mathrm{d} x \mathrm{~d} y=\int_{0}^{\frac{\pi}{2}}\left[\operatorname{areaC}_{1}(\varphi)\right] \mathrm{d} \varphi \\
& =\int_{0}^{\frac{\pi}{2}}\left[\operatorname{areaC}_{1}-\mathrm{A}_{1}(\varphi)\right] \mathrm{d} \varphi=\frac{\pi}{2} \operatorname{areaC}_{1}-\int_{0}^{\frac{\pi}{2}}\left[\mathrm{~A}_{1}(\varphi)\right] \mathrm{d} \varphi .
\end{aligned}
$$

Replacing in the (2.5) the relations (2.6) and (2.7) we obtain

$$
P_{\text {int }}^{(1)}=\frac{2}{\pi a r e a C_{1}} \int_{0}^{\frac{\pi}{2}}\left[A_{1}(\varphi)\right] d \varphi .
$$

Considering formula (2.3) we have

$$
\int_{0}^{\frac{\pi}{2}}\left[A_{1}(\varphi)\right] d \varphi=2(a+b) l-\frac{l^{2}}{2}-\frac{\pi}{2} m^{2} .
$$

The relations (2.1), (2.8) and (2.9) give us the result.

Remark 2.2. For $m=0$ the obstacles become points and the probability $\mathrm{P}_{\text {int }}^{(1)}$ becomes:

So, we find a result of a previous paper [3].

$$
p^{(1)}=\frac{4(a+b) l-l^{2}}{3 \pi a b} .
$$

\section{Obstacles circular sectors}

We consider the lattice $\mathfrak{R}_{2}(a, b ; m)$ with the fundamental cell $C_{2}$ represented in Figure 3 .

By this figure we have that the formula (2.2) is valid for the cell $C_{2}$. Then we have

$$
\operatorname{areaC}_{2}(\varphi)=3 a b-\frac{5 \pi}{4} m^{2}
$$

As in the paragraph 1 , we compute the probability $\mathrm{P}_{\mathrm{int}}^{(2)}$ that a segment $s$ intersects a side of fundamental cell $\mathrm{C}_{2}$. 4

Considering the limiting positions of segment $s$, for a fixed angle $\varphi$, in the cell $\mathrm{C}_{2}$. We obtain the Figure

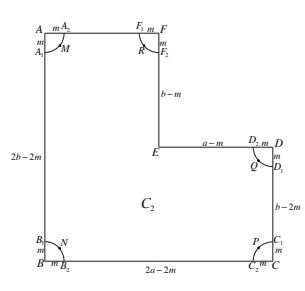

Figure 3

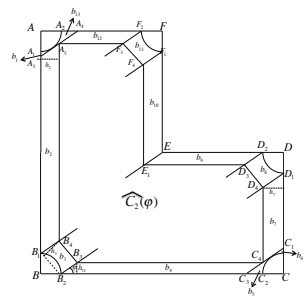

Figure 4

and the formula

$$
\operatorname{area} \widehat{C}_{2}(\varphi)=\operatorname{areaC}_{2}-\sum_{i=1}^{13} \operatorname{areab}_{i}(\varphi)
$$

Theorem 3.1. We have

$$
\mathrm{P}_{\mathrm{int}}^{(2)}=\frac{2\left[2(\mathrm{a}+\mathrm{b}) \mathrm{l}-\frac{\mathrm{l}^{2}}{2}-\frac{\pi \mathrm{m}^{2}(5 \pi-6)}{8}\right]}{\pi\left(3 a b-\frac{5 \pi}{4} \mathrm{~m}^{2}\right)} .
$$

Remark 3.2. For $m=0$ the obstacles become points and the probability $\mathrm{P}_{\text {int }}^{(2)}$ become:

$$
p^{(2)}=\frac{4(a+b) l-l^{2}}{3 \pi a b} .
$$

\section{Obstacles triangular and circular sectors}

We consider the lattice $\mathfrak{R}_{3}(a, b ; m)$ with the fundamental cell $C_{3}$ represented in Figure 5. 
From Figure 5 we have

$$
\operatorname{areaC}_{3}=3 a b-m^{2}-\frac{3 \pi m^{2}}{4}
$$

As in the previous paragraphs, we compute the probability $\mathrm{P}_{\text {int }}^{(3)}$ that a segment $s$ intersects a side of fundamental cell $\mathrm{C}_{3}$.

Considering the limiting positions of segment $s$, for a fixed angle $\varphi$, in the cell $C_{2}$. We obtain Figure 6

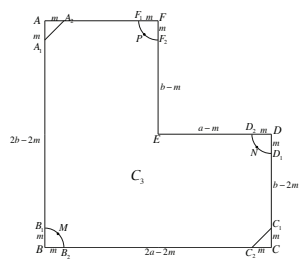

Figure 5

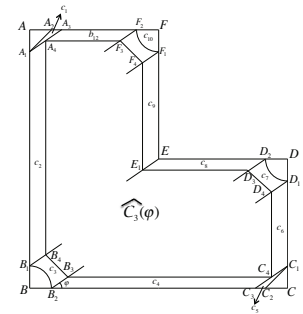

Figure 6

and the formula

$$
\operatorname{area} \widehat{C}_{3}(\varphi)=\operatorname{areaC} C_{3}-\sum_{i=1}^{11} \operatorname{areac}_{i}(\varphi)
$$

Theorem 4.1. We have

$$
P_{i n t}^{(3)}=\frac{4(a+b) l-l^{2}-\frac{\pi(5 \pi-14) m^{2}}{4}}{\pi\left(3 a b-m^{2}-\frac{3 \pi m^{2}}{4}\right)} .
$$

Remark 4.2. If $\mathrm{m}=0$, the obstacles become points and the probability $\mathrm{P}_{\text {int }}$ becomes

$$
p^{(3)}=\frac{4(a+b) l-l^{2}}{3 \pi a b}
$$

Remark 4.3. The relation (2.10), (3.1) and (4.1) give us the evident formula

$$
p^{(1)}=p^{(2)}=p^{(3)} \text {. }
$$

\section{References}

[1] D. Barilla, G. Caristi, A. Puglisi, M. Stoka, A Laplace type problem for two hexagonal lattices of Delone with obstacles, Appl. Math. Sci., 7 (2013), 4571-4581. 1

[2] D. Barilla, G. Caristi, E. Saitta, M. Stoka, A Laplace type problem for lattice with cell composed by two quadrilaterals and one triangle, Appl. Math. Sci., 8 (2014), 789-804. 1

[3] D. Barilla, G. Caristi, A. Puglisi, M. Stoka, Laplace Type Problems for a Triangular Lattice and Different Body Test, Appl. Math. Sci., 8 (2014), 5123-5131. 2

[4] G. Caristi, A. Puglisi, E. Saitta, A Laplace type for an regular lattices with convex-concave cell and obstacles rhombus, Appl. Math. Sci., 7 (2013), 4049-4065. 1

[5] G. Caristi, E. L. Sorte, M. Stoka, Laplace problems for regular lattices with three different types of obstacles, Appl. Math. Sci., 5 (2011), 2765-2773. 1

[6] G. Caristi, M. Stoka, A Laplace type problem for a regular lattice of Dirichlet-Voronoi with different obstacles, Appl. Math. Sci., 5 (2011), 1493-1523. 1

[7] G. Caristi, M. Stoka, A laplace type problem for lattice with axial symmetric and different obstacles type (I), Far East J. Math. Sci., 58 (2011), 99-118. 1

[8] G. Caristi, M. Stoka, A Laplace type problem for lattice with axial symmetry and different type of obstacles (II), Far East J. Math. Sci., 64 (2012), 281-295. 1

[9] A. Duma, M. Stoka, Problems of "Buffon" type for a non-convex lattice, Rend. Circ. Mat. Palermo (2) Suppl., 70 (2002), 237-256. 1

[10] H. Poincarè, Calcul des probabilitès, Gauthier-Villard, Paris, (1912). 2

[11] M. Stoka, Probabilités géométriques de type "Buffon" dans le plan euclidien, Atti Accad. Sci. Torino Cl. Sci. Fis. Mat. Natur., 110 (1976), 53-59. 2 